\title{
Hybrid Lipid/Polymer Nanoparticles for Pulmonary Delivery of siRNA: Development and Fate Upon In Vitro Deposition on the Human Epithelial Airway Barrier
}

\author{
Ivana d'Angelo, $\mathrm{PhD}$, Gabriella Costabile, $\mathrm{PhD}^{2,3}$ Estelle Durantie, $\mathrm{PhD}$, \\ Paola Brocca, $\mathrm{PhD}^{4}$, Valeria Rondelli, $\mathrm{PhD}^{4}$, Annapina Russo, $\mathrm{PhD}^{5}$, Giulia Russo, $\mathrm{PhD}^{5}$ \\ Agnese Miro, PharmD, Fabiana Quaglia, PhD, Alke Petri-Fink, PhD, \\ Barbara Rothen-Rutishauser, $\mathrm{PhD}^{3}$, and Francesca Ungaro, $\mathrm{PhD}^{2}$
}

\begin{abstract}
Background: Nowadays, the downregulation of genes involved in the pathogenesis of severe lung diseases through local siRNA delivery appears an interesting therapeutic approach. In this study, we propose novel hybrid lipid-polymer nanoparticles (hNPs) consisting of poly(lactic-co-glycolic) acid (PLGA) and dipalmitoyl phosphatidylcholine (DPPC) as siRNA inhalation system.

Methods: A panel of DPPC/PLGA hNPs was prepared by emulsion/solvent diffusion and fully characterized. A combination of model siRNAs against the sodium transepithelial channel $(\mathrm{ENaC})$ was entrapped in optimized hNPs comprising or not poly(ethylenimine) (PEI) as third component. siRNA-loaded hNPs were characterized for encapsulation efficiency, release kinetics, aerodynamic properties, and stability in artificial mucus (AM). The fate and cytotoxicity of hNPs upon aerosolization on a triple cell co-culture model (TCCC) mimicking human epithelial airway barrier were assessed. Finally, the effect of siRNA-loaded hNPs on ENaC protein expression at 72 hours was evaluated in A549 cells.

Results: Optimized muco-inert hNPs encapsulating model siRNA with high efficiency were produced. The developed hNPs displayed a hydrodynamic diameter of $\sim 150 \mathrm{~nm}$, a low polydispersity index, a negative $\zeta$ potential close to $-25 \mathrm{mV}$, and a peculiar triphasic siRNA release lasting for 5 days, which slowed down in the presence of PEI. siRNA formulations showed optimal in vitro aerosol performance after delivery with a vibrating mesh nebulizer. Furthermore, small-angle X-ray scattering analyses highlighted an excellent stability upon incubation with AM, confirming the potential of hNPs for direct aerosolization on mucus-lined airways. Studies in TCCC confirmed that fluorescent hNPs are internalized inside airway epithelial cells and do not exert any cytotoxic or acute proinflammatory effect. Finally, a prolonged inhibition of ENaC protein expression was observed in A549 cells upon treatment with siRNA-loaded hNPs.
\end{abstract}

Conclusions: Results demonstrate the great potential of hNPs as carriers for pulmonary delivery of siRNA, prompting toward investigation of their therapeutic effectiveness in severe lung diseases.

Keywords: dipalmitoylphosphatidylcholine, inhalable nanoparticles, poly(ethylenimine), poly(lactic-co-glycolic) acid, siRNA, triple cell coculture

${ }^{1}$ Di.S.T.A.Bi.F., University of Campania “Luigi Vanvitelli," Caserta, Italy.

${ }^{2}$ Laboratory of Drug Delivery, Department of Pharmacy, University of Napoli Federico II, Napoli, Italy.

${ }^{3}$ Adolphe Merkle Institute, University of Fribourg, Fribourg, Switzerland.

${ }_{5}^{4}$ Applied Physics, Department of Medical Biotechnology and Translational Medicine, University of Milano, Milano, Italy.

${ }^{5}$ Laboratory of Biochemistry, Department of Pharmacy, University of Napoli Federico II, Napoli, Italy. 


\section{Introduction}

$\mathbf{R}$ NA INTERFERENCE refers to the inhibition of gene expression by small, double-stranded RNA molecules (small interference RNA or siRNA) that direct the mRNA machinery to degrade a specific mRNA. siRNA therapeutics have many advantages in terms of clinical applications, the first of which is their virtual potential to specifically silence any gene underlying more-or-less complex pathologies. ${ }^{(1,2)}$ In particular, severe lung diseases, such as lung cancer or cystic fibrosis $(\mathrm{CF})$, nowadays represent a very important area of application for siRNA-based therapies. ${ }^{(3)}$ Nevertheless, to harness the full potential of the siRNA strategy for severe lung diseases, an appropriately designed delivery system is mandatory. ${ }^{(4,5)}$

Naked siRNAs have a half-life of less than an hour in human plasma and are often unable to penetrate cell membranes. In fact, cellular uptake and the escape from endolysosomes are critical steps before siRNA reaches the cytoplasm, where its target is located. Conceiving siRNA for local delivery by inhalation, the presence of high mucin, DNA, and actin concentrations, makes airway mucus a very complex barrier to overcome. $^{(4,5)}$ Thus, optimal inhalable formulations are required: (i) to enhance siRNA stability; (ii) to overcome lung cellular and noncellular barriers; and (iii) to increase siRNA availability at the target level.

Amid advanced drug delivery systems, the encapsulation of siRNA into colloidal carriers is considered a very promising formulation approach for inhaled treatment of severe lung diseases. ${ }^{(4,5)}$ In this context, biodegradable poly(lactic-co-glycolic) acid (PLGA) nanoparticles (NPs) are gaining considerable interest since they may provide protection of the therapeutic cargo from enzymatic degradation, prolonged release (i.e., decreased number of administrations), and improved retention in the lungs. ${ }^{(6,7)}$ Nevertheless, particle engineering with excipients aimed to overcome the mucus-lined human lung epithelial barrier is essential. ${ }^{(8,9)}$

In the attempt to combine the most valuable properties of both lipid and polymeric nanocarriers, hybrid lipid-polymer NPs (hNPs) have recently been proposed. ${ }^{(10,11)}$ Thanks to their ability to form a shell upon the polymer core, lipids may be useful to impart peculiar surface properties to the carrier, likely tuning NP interactions with the lung environment, while enhancing their tolerance in the pulmonary tract. ${ }^{(10)}$ In fact, the use of endogenous phospholipids, such as dipalmitoylphosphatidylcholine (DPPC), can be considered a valid approach to increase NP compatibility with the lung environment. Furthermore, DPPC has been shown to improve the technological features of PLGA particles aimed to nucleic acid delivery to the lung. ${ }^{(12,13)}$

Along these lines, the aim of this work was to design and optimize hNPs consisting of PLGA and DPPC, introducing PEI as a third component to assist siRNA encapsulation in the hNPs. A preliminary formulation study allowed to select optimized muco-inert DPPC/PLGA and PEI/DPPC/PLGA hNPs displaying optimal in vitro aerosol performance. A particular attention was devoted to the characterization of the carrier upon contact with simulated lung fluids through a combination of analytical techniques, that is spectrophotometric assay, dynamic light scattering (DLS), and small-angle X-ray scattering (SAXS).

To allow a proof of principle of RNP potential for siRNA delivery to the lung, selected formulations were loaded with model therapeutic siRNAs of interest for CF therapy, which is a combination of siRNA against the $\alpha$ and $\beta$ subunits of the sodium transepithelial channel $(\mathrm{ENaC})$. siRNA-loaded hNPs were fully characterized for size, zeta potential, encapsulation efficiency, and in vitro release kinetics. A thorough in vitro study allowed to assess the fate and the effect of hNPs upon aerosolization on a triple cell co-culture model (TCCC) grown at air-liquid interface (ALI) mimicking the human airway epithelial barrier. ${ }^{(14)}$ Finally, the in vitro effects of siRNA-loaded hNPs on the expression of $\alpha \mathrm{ENaC}$ and $\beta \mathrm{ENaC}$ subunits were evaluated on a model human lung epithelial cell line.

\section{Materials and Methods}

\section{Materials}

Resomer ${ }^{\circledR}$ RG 502H (uncapped PLGA 50:50, inherent viscosity $0.160 .24 \mathrm{dL} / \mathrm{g}$ ) was purchased from Evonik Industries AG (Germany). Rhodamine-labeled PLGA was synthesized as previously described. ${ }^{(15)}$ 1,2-dipalmitoyl-sn-glycero-3phosphocholine (DPPC) was a kind gift of Lipoid $\mathrm{GmbH}$ (Switzerland). siGENOME SMARTpool siRNA against $\alpha$ and $\beta$ subunits of the sodium transepithelial channel (ENaC) (Human SCNN1A and SCNN1B; 19 mer duplexes; Mw = $13.4 \mathrm{kDa}$ ) and siGENOME Non-Targeting siRNA Pool were purchased from Dharmacon (GE Healthcare).

Ph.Eur. grade mannitol (Pearlitol ${ }^{\circledR} \mathrm{C} 160$ ) was kindly gifted by Roquette Italia S.p.a. (Italy). Egg yolk emulsion, deoxyribonucleic acid sodium salt from calf thymus (DNA), diethylenetriaminepentaacetic acid (DTPA), phosphate buffer salts, branched polyethylenimine (PEI; $25 \mathrm{kDa}$ ), potassium chloride, rhodamine B (Rhod-B), RPMI 1640 amino acid solution, sodium chloride, and mucin from porcine stomach were purchased from Sigma-Aldrich (Missouri). Methylene chloride and ethanol $96 \%$ (v/v) were supplied by Carlo Erba (Italy).

\section{Production of DPPC/PLGA hybrid NPS}

DPPC/PLGA hNPs were prepared by an emulsion/solvent diffusion technique. Briefly, a water in oil emulsion (w/o) was achieved adding $100 \mu \mathrm{L}$ of water to $1 \mathrm{~mL}$ of methylene chloride containing PLGA $(10 \mathrm{mg} ; 1 \% \mathrm{w} / \mathrm{v})$ and DPPC at different DPPC/PLGA weight ratios $(1: 10,1: 20,1: 50,1: 100$, and $1: 150)$, under vortex mixing (2400 minutes $^{-1}$; Heidolph, Germany). When needed, PEI was added to the internal water phase at the theoretical loading of $0.016 \mathrm{mg}$ per $100 \mathrm{mg}$ of PLGA. Just after mixing, the w/o emulsion was added to $12.5 \mathrm{~mL}$ of ethanol $96 \%(\mathrm{v} / \mathrm{v})$ under moderate magnetic stirring, leading to immediate nanoparticle precipitation. Then, the formulation was diluted with $12.5 \mathrm{~mL}$ of Milli-Q water under stirring for 10 minutes. Afterward, residue organic solvent was removed by rotary evaporation under vacuum at $30^{\circ} \mathrm{C}$ (Heidolph VV 2000, Germany). hNPs were isolated from the resulting hNP colloidal dispersion $(5 \mathrm{~mL})$ by centrifugation at $7000 \mathrm{rcf}$ for 20 minutes at $4^{\circ} \mathrm{C}$ (Hettich Zentrifugen, Germany) and dispersed in Milli-Q water.

siRNA-loaded hNPs were prepared in optimized formulation conditions at a theoretical loading of $1 \mathrm{nmol} / 100 \mathrm{mg}$ of PLGA (N/P theoretical ratio of 10 in PEI-modified hNPs) by adding siRNA to the internal water phase. 
Fluorescently-labeled hNPs (DPPC/PLGA Rhod $_{\text {and DPPC/ }}$ PLGA $_{\text {Rhod }}$ ) were prepared using rhodamine-labeled PLGA (PLGA-Rhod) in the organic phase at $10 \% \mathrm{w} / \mathrm{w}$ with respect to the total PLGA amount.

\section{Characterization of DPPC/PLGA hybrid NPS}

Size and zeta potential. The hydrodynamic diameter $\left(\mathrm{D}_{\mathrm{H}}\right)$, the polydispersity index $(\mathrm{PI})$, and the zeta potential $(\zeta$ potential) of hNPs were determined by DLS, with a Malvern Zetasizer Nano ZS (Malvern Instruments, United Kingdom). Results are expressed as mean value \pm standard deviation (SD) of triplicate measurements in different batches.

siRNA loading inside hNPs. siRNA actual loading was measured indirectly by quantifying the amount of unencapsulated siRNA. Briefly, just after production, hNPs were collected by centrifugation (7000 rcf for 20 minutes at $4^{\circ} \mathrm{C}$ ) and the supernatant was analyzed for siRNA content using Quant-IT ${ }^{\mathrm{TM}}$ RiboGreen ${ }^{\circledR}$ reagent (Thermo Fisher Scientific, Massachusetts, Stati Uniti) according to the manufacturer's instructions. Quantitative analysis was performed by spectrofluorimetry at $\lambda_{\text {ex }} 480 \mathrm{~nm} / \lambda_{\text {em }} 520 \mathrm{~nm}$ (EnVision ${ }^{\circledR}$ Multilabel Plate Readers, PerkinElmer, Inc.). Results are reported as actual loading (nmol of encapsulated siRNA per $\mathrm{mg}$ of yielded hNPs) and encapsulation efficiency (actual loading/theoretical loading $\times 100) \pm \mathrm{SD}$ of values collected from three different batches.

SAXS spectroscopy. Synchrotron SAXS measurements were performed at the ID02 high-brilliance beamline ${ }^{(16)}$ at the ESRF (Grenoble, France), with a beam cross section of $200 \times 400 \mu \mathrm{m}$ and wavelength $\lambda=0.1 \mathrm{~nm}$, using two different sample detection distances: 1 and $6 \mathrm{~m}$. The range of investigated momentum transfer, $q=(4 \pi / \lambda) \sin (\theta)$, was $0.0116 \mathrm{~nm}-1$ $<\mathrm{q}<6.43 \mathrm{~nm}-1$, where $2 \theta$ is the scattering angle. All measurements were performed at $\mathrm{T}=25^{\circ} \mathrm{C}$. Samples were put in plastic capillaries (KI-BEAM, ENKI srl) with $2 \mathrm{~mm}$ internal diameter, $0.05 \mathrm{~mm}$ wall thickness, and closed with polyethylene caps. Capillaries were then mounted horizontally onto the sample holder. Sample concentration was $10 \mathrm{mg} / \mathrm{mL}$. The exposure time of each measurement was 0.1 seconds, and spectra were checked for radiation damage.

The measured SAXS profiles report the scattered radiation intensity as a function of the momentum transfer, $q$, where $1 \mathrm{~m}$ and $6 \mathrm{~m}$ sample-to-detector distance spectra were merged. Solvent subtraction was obtained by measuring water-filled capillaries and empty capillaries.

In vitro interaction with mucin. The light scattering of hNP aqueous dispersions with or without mucin was assessed by spectrophotometry as previously described. ${ }^{(8,17)}$ Briefly, a saturated solution of type II mucin was prepared by centrifugation at $6000 \mathrm{rcf}$ for 20 minutes in a mucin dispersion in water $(0.08 \% \mathrm{w} / \mathrm{v})$ stirred overnight. Then, hNPs were dispersed in the mucin solution at a concentration of $1 \mathrm{mg} / \mathrm{mL}$ by vortexing for 1 minute. The light scattering of the mucin/ hNPs mixtures was measured by spectrophotometric analysis at $650 \mathrm{~nm}$ at time 0 and after incubation for 30 and 60 minutes at room temperature. Reference absorbances of mucin and $1 \mathrm{mg} / \mathrm{mL} \mathrm{hNP}$ dispersions in water were also evaluated.
Experiments were run in triplicate and results are expressed as absorbance at $650 \mathrm{~nm} \pm \mathrm{SD}$ over time.

The interactions of hNPs with mucin were further investigated by DLS as previously reported. ${ }^{(9)}$ Briefly, the hNP dispersions in mucin were examined with the Malvern Zetasizer Nano ZS (Malvern Instruments, United Kingdom). Experiments were run in triplicate and results are reported as representative size distribution by intensity of hNPs in mucin versus hNPs in water. The size distribution profile of the mucin solution was also recorded as control.

Particle stability in artificial mucus. To assess the stability of siRNA-loaded hNPs and their ability to diffuse inside airway mucus, SAXS analyses were performed on hNP dispersions in the presence of artificial mucus (AM). As previously reported, ${ }^{(8)} \mathrm{AM}$ was prepared by adding $25 \mu \mathrm{L}$ of sterile egg yolk emulsion, $25 \mathrm{mg}$ of mucin, $20 \mathrm{mg}$ of DNA, $30 \mu \mathrm{L}$ of aqueous DTPA $(1 \mathrm{mg} / \mathrm{mL}), 25 \mathrm{mg} \mathrm{NaCl}, 11 \mathrm{mg} \mathrm{KCl}$, and $100 \mu \mathrm{L}$ of RPMI 1640 to $5 \mathrm{~mL}$ of water. The dispersion was stirred until a homogenous mixture was obtained. For stability studies, aqueous dispersions of siRNA-loaded hNPs $(10 \mathrm{mg} /$ $\mathrm{mL}$ ) were mixed with $\mathrm{AM}$ in a 1:3 volume ratio for a total $40 \mu \mathrm{L}$ sample volume. After a 1-hour equilibration time, SAXS was performed as described above. Particle structure after a 12-hour incubation with AM was verified by repeated SAXS measurements.

In vitro release kinetics of siRNA. siRNA-loaded hNPs were characterized for in vitro siRNA release kinetics in phosphate buffer $(120 \mathrm{mM} \mathrm{NaCl}, 2.7 \mathrm{mM} \mathrm{KCl}$, and $10 \mathrm{mM}$ Na2HPO4) at pH 7.2 (phosphate-buffered saline [PBS]). Release studies were performed upon dilution of hNP dispersions in PBS to a theoretical siRNA concentration of $0.2 \mathrm{nmol} / \mathrm{mL}$. hNP dispersions were incubated in a horizontal shaking water bath at $37^{\circ} \mathrm{C}$ (ShakeTemp SW 22, Julabo, Italy). At scheduled time intervals, samples were centrifuged at $7000 \mathrm{rcf}$ for 20 minutes at $4^{\circ} \mathrm{C}$ to isolate hNPs and the release medium was withdrawn and analyzed for siRNA content by spectrofluorimetric analysis as described for actual loading. The medium was replaced by the same amount of fresh PBS. Experiments were carried out in triplicate and results expressed as the percentage of siRNA released \pm SD.

In Vitro aerosol performance. The aerosolization prop-

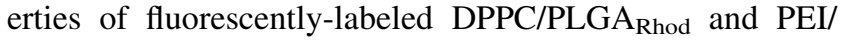
$\mathrm{DPPC}_{\mathrm{PLGA}} \mathrm{Rhod}_{\mathrm{hNP}}$ dispersions were investigated in vitro after delivery from the Aeroneb ${ }^{\circledR}$ Go nebulizer (Aerogen Ltd., Ireland) by a Next Generation Impactor (NGI) (Copley Scientific, United Kingdom) according to the Comité Européen de Normalization (CEN) standard methodology for nebulizer systems with sampling at $15 \mathrm{~L} /$ minutes and insertion of a filter in micro-orifice collector (MOC). ${ }^{(18)}$ Briefly, $1 \mathrm{~mL}$ of $\mathrm{hNP}$ dispersion was transferred to the reservoir of the nebulizer, which was connected to the induction port of the NGI. The nebulizer was operated at $15 \mathrm{~L} /$ minutes, and the aerosol was drawn through the impactor for 5 minutes, until dryness.

The amount of fluorescent hNPs remaining inside the nebulizer chamber, deposited on the seven NGI collection cups, and in the induction port was quantitatively recovered in $\mathrm{NaOH} 0.5 \mathrm{M}$. After stirring at room temperature for 1 hour, the amount of fluorescent hNPs in the resulting solutions was determined by spectrofluorimetric analysis at $\lambda_{\mathrm{ex}}$ 
$553 \mathrm{~nm} / \lambda_{\mathrm{em}} 577 \mathrm{~nm}$ (GloMax ${ }^{\circledR}$ Explorer, Promega, Italy) as previously reported. ${ }^{(8)}$ Calibration curves were derived by analyzing serial dilutions of DPPC/PLGA Rhod $_{\text {Or PEI/DPPC/ }}$ PLGA $_{\text {Rhod }}$ standard solutions prepared from a stock of hNPs degraded in $\mathrm{NaOH} 0.5 \mathrm{M}$. The linearity of the response was verified over the concentration range $2-200 \mu \mathrm{g} / \mathrm{mL}\left(r^{2} \geq\right.$ 0.999). Each experiment was run in triplicate.

The emitted dose (ED) was measured as the difference between the total amount of hNPs initially placed and the amount remaining in the nebulizer chamber. Upon emission, the experimental mass median aerodynamic diameter $\left(\mathrm{MMAD}_{\text {exp }}\right)$ and the geometric SD (GSD) were calculated according to the European Pharmacopoeia, deriving a plot of cumulative mass of particle retained in each collection cup (expressed as percent of total mass recovered in the impactor) versus the cutoff diameter of the respective stage.

The fine particle fraction (FPF) was calculated taking into account the amount of hNPs deposited in stages 3-7 (MMA$\mathrm{D}_{\exp }<5.39 \mu \mathrm{m}$ ) compared to the initial amount loaded into the nebulizer chamber, while the respirable fraction (RF) referred to the total amount recovered from the NGI.

\section{In vitro studies on airway triple cell cocultures}

All in vitro exposure experiments were conducted with a three-dimensional TCCC mimicking the human epithelial airway barrier comprising human bronchial epithelial cells (16HBE14o-), human blood monocyte-derived macrophages (MDM), and dendritic cells (MDDC). ${ }^{(14)}$

16HBE140- cell cultures. 16HBE14o- cells were kindly provided by Dieter Gruenert (passage number P2.54; University California, San Francisco, CA). Cells were maintained in minimum essential media $1 \times$ (with Earle's Salts, $25 \mathrm{mM}$ HEPES and without L-glutamine; Gibco BRL, Thermo Fisher Scientific), supplemented with $1 \%$ L-glutamine, $1 \%$ penicillin/streptomycin, and $10 \%$ fetal calf serum.

For experimental cultures, cells were seeded at a density of $0.5 \times 10^{6}$ cells/insert on transparent BD Falcon (Corning) cell culture inserts (surface area of $0.9 \mathrm{~cm}^{2}$, pores with $3.0 \mu \mathrm{m}$ diameter, and PET membranes for 12-well plates). The cell culture inserts were pretreated with $150 \mu \mathrm{L}$ of a fibronectin coating solution containing $0.1 \mathrm{mg} / \mathrm{mL}$ bovine serum albumin (Sigma-Aldrich), 1\% Type I bovine collagen (BD Biosciences, Becton Dickinson, New Jersey), and 1\% human fibronectin (BD Biosciences, Becton Dickinson) in the LHC Basal Medium (Sigma-Aldrich). Inserts were placed in BD Falcon tissue culture plates (12-well plates) with $0.5 \mathrm{~mL}$ medium in the upper and $1.5 \mathrm{~mL}$ in the lower chamber. The cells were kept at $37^{\circ} \mathrm{C}$ in $5 \% \mathrm{CO}_{2}$ humidified atmosphere for 7 days (medium changed after $3-4$ days).

TCCC system. To produce the TCCC in vitro model, 16HBE14o- cell cultures were supplemented on the apical side with MDM and on the basolateral side with MDDC derived from human blood as previously reported. ${ }^{(14,19)}$ Briefly, 16HBE14o- cells were cultured as described above. At 5 days, the medium was removed from the upper and lower chamber, the inserts turned upside down, and the bottom was abraded carefully with a cell scraper. The inserts were then incubated with $65 \mu \mathrm{L}$ medium containing $83 \times 10^{4} \mathrm{MDDC} / \mathrm{mL}$ on the basal side for 2 hours. Afterward, the inserts were turned around again and placed in BD Falcon (Corning) tissue culture plates (12-well plates). In the lower chamber, $1.5 \mathrm{~mL}$ of medium, and at the apical side of the TCCC, $500 \mu \mathrm{L}$ medium containing $2.5 \times 10^{4} \mathrm{MDM} / \mathrm{mL}$ were added. The complete TCCC systems were kept with $1.5 \mathrm{~mL}$ of medium in the lower chamber and $0.5 \mathrm{~mL}$ in the upper chamber at $37^{\circ} \mathrm{C}$ in $5 \% \mathrm{CO}_{2}$ humidified atmosphere for 24 hours.

To establish the ALI, the medium in the upper chamber of the TCCC systems was removed 24 hours before exposure in the Vitrocell ${ }^{\circledR}$ Cloud, while the medium in the lower chamber was replaced by $0.6 \mathrm{~mL}$ of fresh medium.

In vitro TCCC exposure to aerosolized NPs. Cell exposure to hNPs at ALI was carried out with the Vitrocell Cloud system (Vitrocell Systems GmbH, Germany). Briefly, the Vitrocell Cloud consists of three main components: a nebulizer, an exposure chamber, and a flow system, with an incubation chamber providing suitable temperature and humidity cell culture conditions. The aerosol is generated into the exposure chamber by a perforated vibrating membrane nebulizer (AeronebPro, with a span of $2.5-6.0 \mu \mathrm{m}$ volume mean diameter). Before each experiment, freeze-dried hNPs were dispersed in $0.5 \mathrm{mM}$ sodium chloride.

For TCCC exposure to low hNP concentrations, $200 \mu \mathrm{L}$ of a $0.5 \mathrm{mg} / \mathrm{mL} \mathrm{hNP}$ dispersion was aerosolized $(0.1 \mathrm{mg}$ of hNPs). For high hNP concentrations, TCCC were exposed to further $400 \mu \mathrm{L}(2 \times 200 \mu \mathrm{L})$ of a $0.9 \mathrm{mg} / \mathrm{mL} \mathrm{hNP}$ dispersion ( $0.5 \mathrm{mg}$ of hNPs). TCCC exposed to $0.5 \mathrm{mM}$ sodium chloride and mannitol aqueous solutions was used as controls. Following the exposure in the chamber (10 and 30 minutes for 0.1 and $0.5 \mathrm{mg}$ of hNPs, respectively), the inserts were immediately placed in new BD Falcon 12- well tissue culture plates containing $0.6 \mathrm{~mL}$ of fresh medium for cell uptake studies, cytotoxicity assay, and tumor necrosis factor alpha (TNF- $\alpha$ ) release. The morphology of hNPs was analyzed by transmission electron microscopy (TEM) before nebulization and after exposing a 400 mesh copper film grid to nebulized hNPs. The analysis was performed with a FEI Tecnai spirit TEM at $120 \mathrm{kV}$ and images were acquired with a Veleta CCD camera $(2048 \times 2048$ pixels $)$.

Nanoparticle deposition efficiency. The amount of hNPs deposited upon nebulization inside the Vitrocell Cloud was assessed. To this purpose, glass coverslips $(24 \times 24 \mathrm{~mm})$ were placed onto the compartments for cell culture inserts and exposed to different amounts of aerosolized rhodamine-labeled DPPC/PLGA Rhod $_{\text {and PEI/DPPC/PLGA }}$ Rhod hNPs. After the exposure, coverslips were removed and rinsed with $\mathrm{NaOH}$ $0.5 \mathrm{M}(500 \mu \mathrm{L})$. After stirring at room temperature for 1 hour, the amount of fluorescent hNPs in the solutions was quantified by spectrofluorimetric analysis as described for in vitro aerosol performance. Results are reported as $\mu \mathrm{g}$ of hNPs deposited per $\mathrm{cm}^{2} \pm \mathrm{SD}$ of values collected from triplicate analyses.

Cell uptake studies. The TCCC was exposed to different amounts of aerosolized rhodamine-labeled DPPC/PLGA Rhod

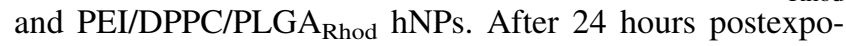
sure, TCCC were fixed for 15 minutes with 3\% paraformaldehyde in PBS at room temperature and cells were washed thrice in PBS. The F-actin cytoskeleton and the DNA of all cells were stained with phalloidin (ALEXA 488) (Molecular Probes, Life Technologies Europe B.V., Switzerland) and 
4',6- diamidin-2-phenylindol (DAPI) $(1 \mu \mathrm{g} / \mathrm{mL})$ in a $1: 100$ and $1: 50$ dilution in $0.3 \%$ Triton $\mathrm{X}-100$ in PBS, respectively. For microscopy, membranes were embedded in Glycergel (DAKO Schweiz AG, Baar, Switzerland). Sample analysis was performed with an inverted Zeiss confocal laser scanning microscope 710 (LSM, Axio Observer.Z1, Zeiss, Germany). Representative images (z-stacks) were recorded at three independent fields of view for each sample. Image processing was achieved using the 3D reconstruction software Imaris (Bitplane AG, Switzerland).

Cytotoxicity assay. The TCCC was exposed to different amounts of aerosolized siRNA-DPPC/PLGA and siRNA-PEI/ DPPC/PLGA, and corresponding empty hNPs. After 24 hours postexposure at $37^{\circ} \mathrm{C}$, lactate dehydrogenase $(\mathrm{LDH})$ release by the TCCC, indicative of cell membrane damage, was assessed by the LDH cytotoxicity detection kit (Roche Applied Science, Germany) according to the manufacturer's guidelines. The test was performed in triplicates and evaluated in comparison to the negative controls, which are TCCC exposed to aqueous solutions of $0.5 \mathrm{mM}$ sodium chloride or mannitol at the same concentrations of hNP dispersions. As a positive control, TCCC were treated with $100 \mu \mathrm{L}$ of $0.2 \%$ Triton X-100 in PBS on the apical side and incubated for 24 hours at $37^{\circ} \mathrm{C}$ and $5 \% \mathrm{CO}_{2}$.

Tumor necrosis factor- $\alpha$ release. The potential proinflammatory response was also investigated after 24 hours postexposure to hNPs by quantifying the release of TNF- $\alpha$ using an ELISA kit (R\&D Systems) according to the manufacturer's protocol.

\section{In vitro gene silencing effect}

Cell cytotoxicity. A549 cells were seeded onto 96-well plates $\left(5 \times 10^{4}\right.$ cells/well) and incubated with hNPs for 24 and 48-72 hours. Then, cell viability was evaluated by MTT assay as previously reported. ${ }^{(20)}$

Cell culture, transfection, and treatment. Human alveolar basal epithelial cell line (A549) was cultured in Dulbecco's modified Eagle's medium supplemented with $10 \%$ heatinactivated fetal bovine serum (Microtech), $1.5 \mathrm{mM} \mathrm{L}$ glutamine, 100 units $/ \mathrm{mL}$ penicillin, and $100 \mu \mathrm{g} / \mathrm{mL}$ streptomycin under humidified atmosphere of $5 \% \mathrm{CO}_{2}$ at $37^{\circ} \mathrm{C}$. siRNA transfections and treatments were performed as previously described. ${ }^{(20 b)}$

Western blotting. Total proteins $(75 \mu \mathrm{g})$ were extracted from the cells, separated on $10 \%$ sodium dodecyl sulfatepolyacrylamide gel electrophoresis and electrotransferred on polyvinylidene fluoride (PVDF) as previously described. ${ }^{(21)}$ Then, the membrane was incubated for 2 hours with $5 \%$ nonfat milk to block nonspecific binding sites and incubated 2 hours at room temperature with the following antibodies: anti- $\beta \mathrm{ENaC}$ (Santa Cruz Biotechnology), antiSCNN1A (Proteintech Europe), and anti- $\beta$-actin (Santa Cruz Biotechnology). Densitometric analysis was carried out using ImageJ software (Image $1.49 \mathrm{v}$, available on: http://imagej. nih.gov/ij, National Institutes of Health, Bethesda, MD).

\section{Data analysis}

Cell studies were performed in triplicate and results are expressed as mean value \pm standard error mean $(n=3)$. The significance of differences was determined with the software Kaleida graph using one-way analysis of variance followed by Bonferroni post-hoc test. A $p$-value $<0.05$ was considered significant.

\section{Results}

\section{Development studies of DPPC/PLGA hNPs for siRNA loading}

In-depth formulation studies were performed on empty hNPs to establish the optimal ratio between the selected components, that is, PLGA, DPPC, and PEI. Overall hNP properties are reported in Table 1.

Independent of the amount of DPPC added to the organic phase and the presence of PEI in the internal water phase, the adopted formulation conditions lead to the formation of hNPs with $\mathrm{D}_{\mathrm{H}}$ ranging between $\sim 135$ and $\sim 169 \mathrm{~nm}$, low PI $(<0.130)$, and negative $\zeta$ potentials (between -16 and $-30 \mathrm{mV})$. Nevertheless, in each case, a flocculation of the exceeding amount of the lipid component was apparent after rotary evaporation of the diffusion solvent under vacuum.

This phenomenon was particularly evident when high amounts of DPPC were added to the formulation (i.e., DPPC/

Table 1. Composition, Size, Polydispersity Index, and Zeta Potential of Empty DPPC/PLGA AND PEI/DPPC/PLGA Hybrid LiPID-Polymer NANOPARTICles

\begin{tabular}{|c|c|c|c|c|c|}
\hline Formulation & $\begin{array}{c}\text { PEI theoretical } \\
\text { loading (mg/100 mg } \\
\text { of PLGA) }\end{array}$ & $\begin{array}{c}D P P C / P L G A \\
\text { ratio }(w / w)\end{array}$ & $\begin{array}{c}D_{H} \\
(n m \pm S D)\end{array}$ & $\begin{array}{c}P I \\
(m e a n \pm S D)\end{array}$ & $\begin{array}{l}\zeta \text { potential } \\
(m V \pm S D)\end{array}$ \\
\hline DPPC/PLGA10 & - & $1: 10$ & $151.3 \pm 21.3$ & $0.127 \pm 0.079$ & $-25.3 \pm 0.5$ \\
\hline DPPC/PLGA 20 & - & $1: 20$ & $149.7 \pm 2.1$ & $0.083 \pm 0.006$ & $-23.2 \pm 2.4$ \\
\hline DPPC/PLGA 50 & - & $1: 50$ & $135.4 \pm 17.2$ & $0.088 \pm 0.008$ & $-24.3 \pm 6.0$ \\
\hline DPPC/PLGA 100 & - & $1: 100$ & $158.9 \pm 7.1$ & $0.082 \pm 0.004$ & $-28.4 \pm 2.9$ \\
\hline DPPC/PLGA 150 & - & $1: 150$ & $168.6 \pm 7.1$ & $0.124 \pm 0.094$ & $-15.7 \pm 1.3$ \\
\hline PEI/DPPC/PLGA 10 & 0.016 & $1: 10$ & $166.5 \pm 13.9$ & $0.128 \pm 0.093$ & $-26.1 \pm 0.5$ \\
\hline PEI/DPPC/PLGA 20 & 0.016 & $1: 20$ & $165.4 \pm 9.1$ & $0.102 \pm 0.006$ & $-31.7 \pm 1.2$ \\
\hline PEI/DPPC/PLGA 50 & 0.016 & $1: 50$ & $162.3 \pm 13.9$ & $0.124 \pm 0.041$ & $-28.0 \pm 0.7$ \\
\hline PEI/DPPC/PLGA 100 & 0.016 & $1: 100$ & $150.9 \pm 30.4$ & $0.106 \pm 0.027$ & $-28.0 \pm 0.3$ \\
\hline PEI/DPPC/PLGA 150 & 0.016 & $1: 150$ & $146.4 \pm 0.3$ & $0.123 \pm 0.027$ & $-22.2 \pm 2.7$ \\
\hline
\end{tabular}

PI, polydispersity index; SD, standard deviation. 

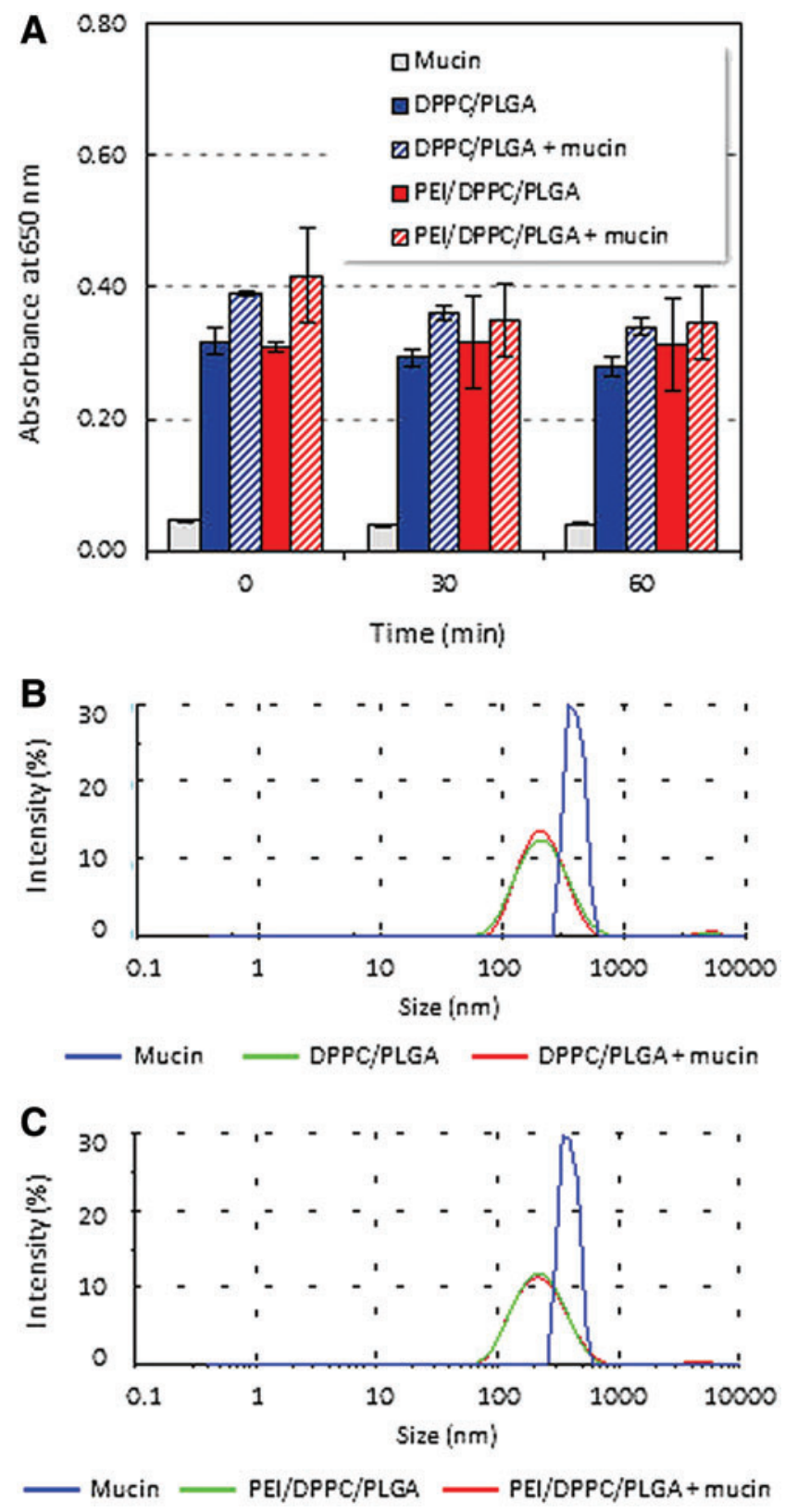

FIG. 1. In vitro assessment of mucin interactions with optimized empty DPPC/PLGA and PEI/DPPC/PLGA hNPs. (A) Scattering at $650 \mathrm{~nm}$ of hNPs in mucin $(1 \mathrm{mg} / \mathrm{mL})$ over time. The absorbance at $650 \mathrm{~nm}$ of mucin and hNP dispersions in water is reported as control. (B-C) Size distribution by intensity of hNP dispersions in water or a saturated mucin solution evaluated by DLS. The size distribution profile of mucin in water is reported as control (B DPPC/PLGA hNPs and C PEI/ DPPC/PLGA). hNPs, hybrid lipid-polymer nanoparticles. Color images available online at www.liebertpub.com/jamp

PLGA 1:10 w/w), but it was limited up to a theoretical DPPC/ PLGA ratio of 1:20. Thus, this last ratio was selected for further studies to maximize the amount of DPPC associated to the NPs, while limiting flocculation. The corresponding formulations, from here on, are named DPPC/PLGA and PEI/DPPC/PLGA.

Mucin/nanoparticle interaction studies were performed by measuring the absorbance at $650 \mathrm{~nm}$ of DPPC/PLGA and PEI/DPPC/PLGA hNPs in mucin over time (Fig. 1A). While the absorbance of the reference mucin dispersion was close to zero, higher values of absorbance were observed at each time point upon addition of hNP dispersions. Nevertheless, no significant differences were observed between absorbance of hNPs dispersed in water or mucin (Fig. 1A). The interactions between mucin and either DPPC/PLGA or PEI/DPPC/PLGA hNP were analyzed also by DLS (Fig. 1B, C, respectively).

The combined particle size distribution for the various replicates shows no change in the position and intensity of the peak. In line with previous studies, ${ }^{(9)}$ the apparent dimension of bare PLGA is significantly shifted to larger sizes upon addition of mucin, reflecting strong mucin/NP hydrophobic interactions, which likely provide a basis for mucoadhesive NPs (Supplementary Fig. S1A; Supplementary Data are available online at www.liebertpub.com/jamp). A similar behavior was observed for bare PEI-PLGA NPs (Supplementary Fig. S1B).

\section{Characterization of siRNA-loaded DPPC/PLGA hNPs}

A model siRNA against ENaC was entrapped in DPPC/ PLGA and PEI/DPPC/PLGA formulations at the theoretical loading of $1 \mathrm{nmol} / 100 \mathrm{mg}$ of PLGA. The addition of siRNA inside the formulation did not result in significant changes of hNP size and PI (Table 2) compared to empty hNPs (Table 1). siRNA-loaded hNPs with a $\mathrm{D}_{\mathrm{H}}$ around $140 \mathrm{~nm}(141$ and $142 \mathrm{~nm}$ for siRNA-DPPC/PLGA and siRNA-PEI/DPPC/PLGA, respectively) and low PI were produced with good yields (52\% and $53 \%$ for siRNA-DPPC/PLGA and siRNA-PEI/DPPC/ PLGA, respectively). siRNA was effectively incorporated inside DPPC/PLGA hNPs with a mean encapsulation efficiency of $75 \%$. The addition of PEI inside the formulation allowed to reach an excellent encapsulation efficiency of $96 \%$, thus increasing siRNA actual loading from 1.35 to $1.74 \mathrm{nmol}$ per $100 \mathrm{mg}$ of hNPs.

Results of in vitro release studies performed at physiological $\mathrm{pH}$ and temperature $\left(\mathrm{pH} 7.2\right.$ and $\left.37^{\circ} \mathrm{C}\right)$ are reported in Figure 2 as percentage of siRNA released over time.

Both siRNA-DPPC/PLGA and siRNA-PEI/DPPC/PLGA hNPs displayed a typical triphasic release profile, characterized by an initial burst, with more than $50 \%$ of siRNA released in the first hours, followed by a slow release phase lasting a couple of days and a final fast release time period after 4-5 days. Although, both formulations show similar release profile, siRNA/

Table 2. Properties of SiRNA-Loaded Hybrid LiPID-POLYMER NANOPARTICLES

\begin{tabular}{lcc}
\hline & siRNA-DPPC/ & siRNA-PEI/ \\
& PLGA & DPPC/PLGA \\
\hline $\mathrm{D}_{\mathrm{H}}(\mathrm{nm} \pm \mathrm{SD})$ & $141 \pm 0.50$ & $142 \pm 1.8$ \\
$\mathrm{PI}(\mathrm{mean} \pm \mathrm{SD})$ & $0.120 \pm 0.018$ & $0.114 \pm 0.0050$ \\
$\zeta$ potential $(\mathrm{mV} \pm \mathrm{SD})$ & $-29.1 \pm 1.6$ & $-29.9 \pm 1.4$ \\
Yield of production & $52.0 \pm 8.9$ & $53.4 \pm 2.5$ \\
$\quad(\% \pm \mathrm{SD})$ & $1.35 \pm 0.02$ & $1.74 \pm 0.04$ \\
$\begin{array}{l}\text { siRNA actual loading } \\
\quad(\% \pm \mathrm{SD})\end{array}$ & & \\
$\begin{array}{l}\text { Encapsulation efficiency } \\
\quad(\% \pm \mathrm{SD})\end{array}$ & $74.8 \pm 1.5$ & $95.9 \pm 2.2$ \\
\hline
\end{tabular}

${ }^{a}$ siRNA loading was expressed as nmol of encapsulated siRNA per $100 \mathrm{mg}$ of hNPs. siRNA theoretical loading was $1 \mathrm{nmol} / 100 \mathrm{mg}$ of hNPs.

hNPs, hybrid lipid-polymer nanoparticles. 


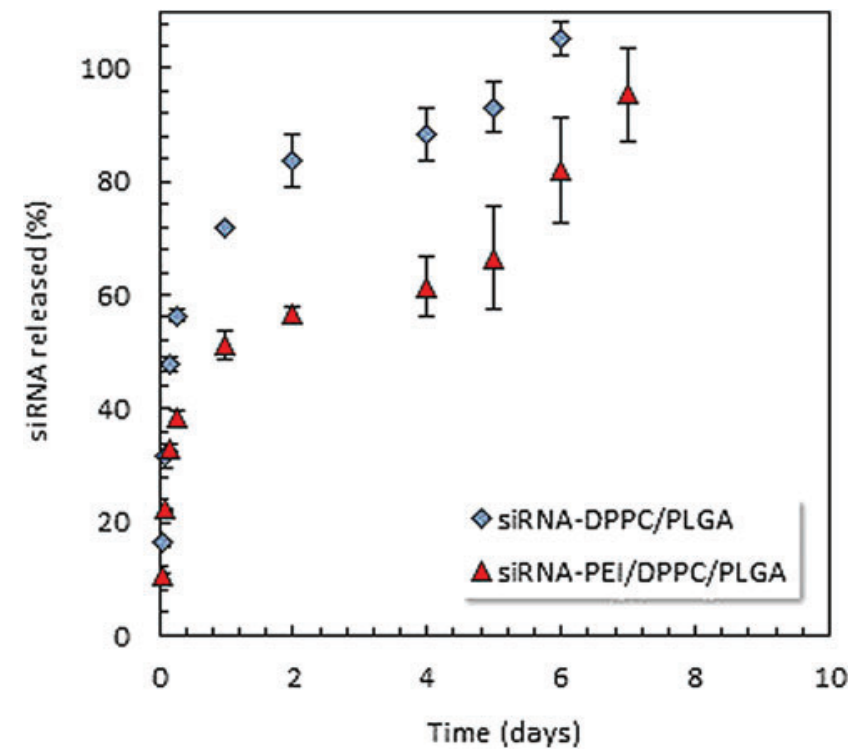

FIG. 2. In vitro release kinetics of siRNA from hNPs in PBS at $\mathrm{pH}$ 7.2. Data are expressed as siRNA released $(\%) \pm \mathrm{SD}$ $(n=3)$. PBS, phosphate-buffered saline; SD, standard deviation. Color images available online at www.liebertpub.com/ jamp

DPPC/PLGA hNPs provided a significantly more rapid release compared to siRNA-PEI/DPPC/PLGA hNPs. Nevertheless, both hNP formulations sustained siRNA release for 1 week.

The I(q) SAXS spectra of siRNA-DPPC/PLGA and siRNAPEI/DPPC/PLGA hNPs are reported in Figure 3 (Top panel). A low $q$ region affected by the finite size of the particle in the range of the $100 \mathrm{~nm}$ distances can be identified. At higher q, an extended region with q-4 slope shows up, which identifies a sharp solvent-particle interface and a smooth particle surface. Giunier analysis $^{(22)}$ in the interval $0.0116 \mathrm{~nm}^{-1}<\mathrm{q}<0.0246 \mathrm{~nm}^{-1}$ gives gyration radii $\mathrm{Rg}=53 \pm 0.5 \mathrm{~nm}$ and $\mathrm{Rg}=52 \pm 0.6 \mathrm{~nm}$ for siRNA-DPPC/PLGA and siRNA-PEI/DPPC/PLGA hNPs, respectively. If modeled as spheres, this corresponds to particle radii of 68 and $67 \mathrm{~nm}$, in good agreement with hydrodynamic radii (Table 2 ). The best fit of $\mathrm{I}(\mathrm{q})$ is obtained by applying the form factor of a distribution of polydispersed spheres with a number-averaged mean radius of $45 \pm 9 \mathrm{~nm}$ for siRNA-DPPC/ PLGA and $42 \pm 11 \mathrm{~nm}$ for siRNA-PEI/DPPC/PLGA hNPs. The fits are shown in Figure 3 (red lines).

The phospholipid component is possibly coating the PLGA particle, without forming a bilayer envelope. A comparison of the spectra of bare PLGA NPs and DPPC/PLGA hNPs is reported in Supplementary Figure S2 to better illustrate this point. We underline that PEI and siRNA contents are actually of the order of $\mu \mathrm{g}$ over $10 \mathrm{mg}$ of particles, so that scattering contribution from those components are not detectable.

Applying SAXS analysis, the stability of hNPs in mucus was studied during 12 hours after mixing AM with aqueous dispersions of hNPs in a 3:1 volume ratio. The scattering spectra obtained after equilibration over 12 hours are reported in Figure 3 (Bottom panel). Of note, the obtained spectrum is consistent with the sum of the naked particle spectrum and the mucus spectrum that give the reconstructed line shown in the figure (blue line).

In vitro aerosol performance of the optimized hNP formulations was first assessed through NGI. To easily quantify

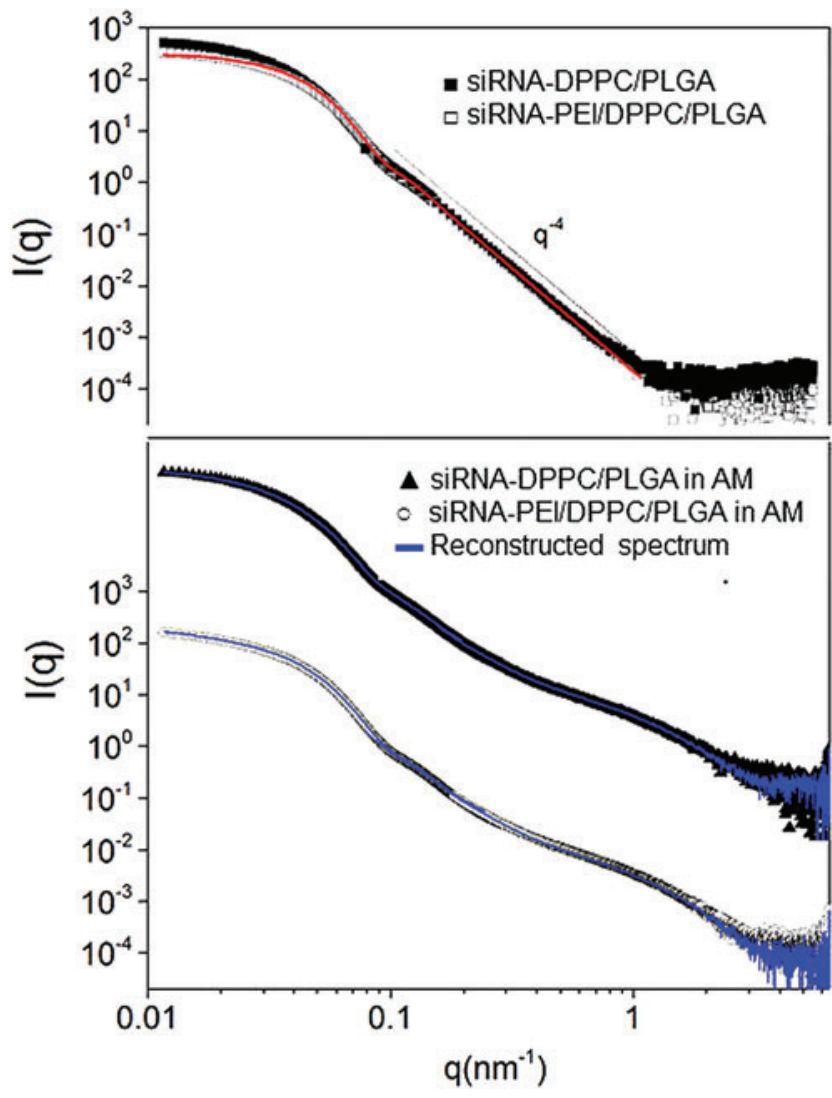

FIG. 3. Small-angle X-ray scattering spectra of siRNAloaded hNPs. Top panel: siRNA-DPPC/PLGA (full dots) and siRNA-PEI/DPPC/PLGA (empty dots) in water. The red line is the representative fitting of the siRNA-PEI/ DPPC/PLGA data with the form factor of a polydisperse sphere (red line). Bottom panel: siRNA-DPPC/PLGA (top) and siRNA-PEI/DPPC/PLGA (bottom) after 12 hours of incubation with artificial mucus (AM) (empty dots) versus a reconstructed spectrum obtained by summing up AM and naked hNP spectra (blue line). siRNA-DPPC/PLGA spectrum is stacked for clarity. Color images available online at www.liebertpub.com/jamp

the amount of hNPs deposited in each cup, blank fluorescent DPPC/PLGA $_{\text {Rhod }}$ and PEI/DPPC/PLGA Rhod $_{\text {hNPs were used }}$ (Fig. 4). In fact, neither the presence of siRNA nor the addition of PLGA-Rhod affected hNP size and surface properties, which are crucial for aerosolization.

According to the new regulatory guidance, both USP and Ph.Eur. recognize the suitability of the NGI for nebulizer characterization when used at $15 \mathrm{~L} / \mathrm{min}$ excluding the preseparator and placing an internal filter below the MOC. ${ }^{(18)}$ In this configuration, the seven NGI stages produce cutoff diameters in the range $14.1-0.98 \mu \mathrm{m}$, while the last five stages have cutoff diameters between 5.39 and $0.98 \mu \mathrm{m}$. Figure $4 \mathrm{~A}$ describes the cumulative droplet size distribution of the hNP dose emitted after aerosolization from an Aeroneb Go vibratingmesh nebulizer. In both cases, the best fit line was from logarithmic linear regression $\left(r^{2}>0.96\right)$. The deposition patterns of DPPC/PLGA $A_{\text {Rhod }}$ and PEI/DPPC/PLGA Rhod $_{\text {throughout the }}$ NGI cups are shown in Figure 4B. In both cases, less than $1.5 \%$ of the ED of hNPs was deposited in the throat, with $65 \%-75 \%$

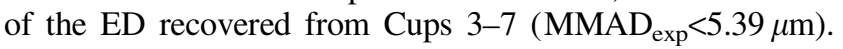



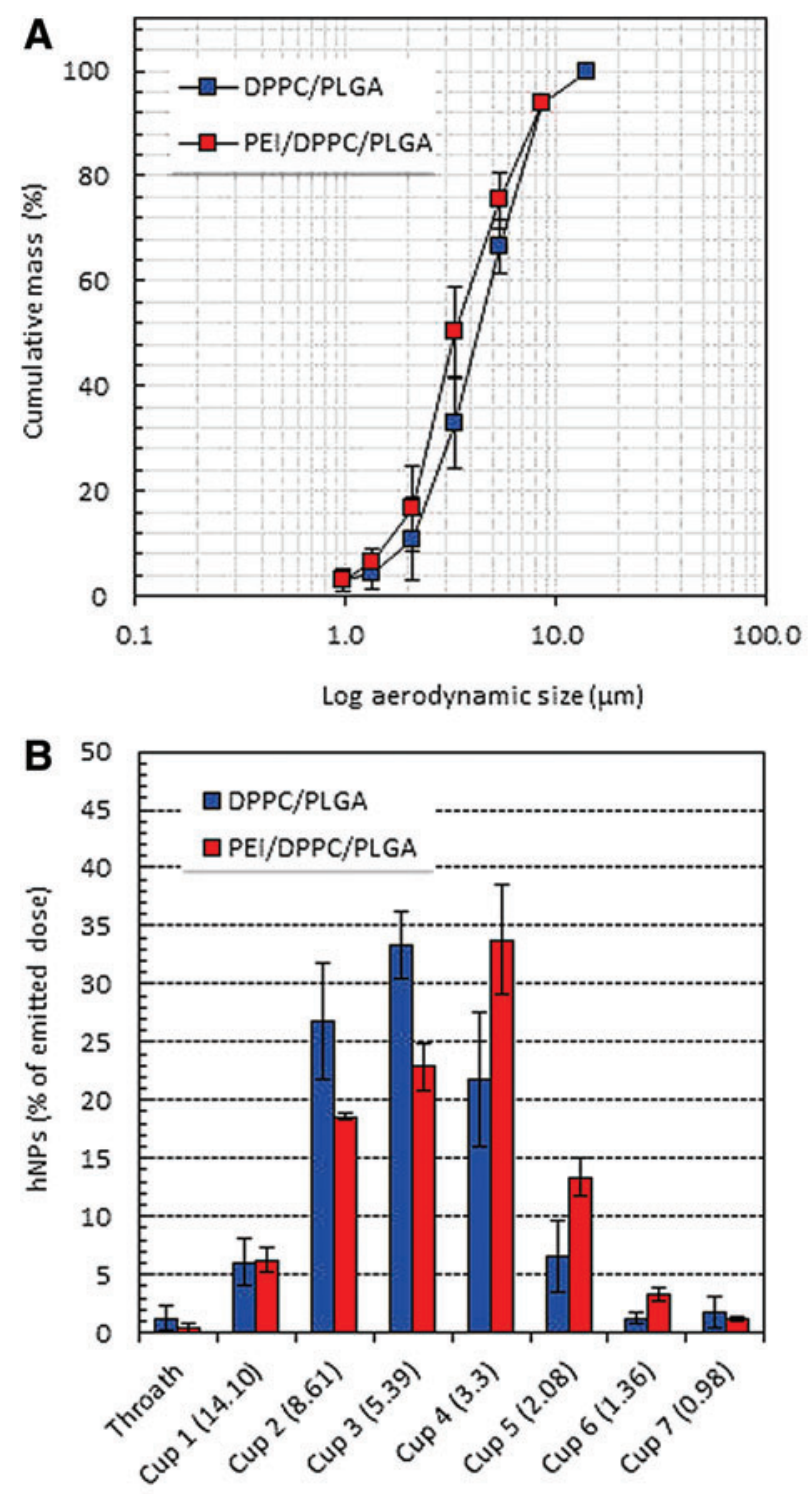

C

\begin{tabular}{|c|c|c|}
\hline & DPPC/PLGA & $P E 1 / D P P C / P L G A$ \\
\hline$M M_{\exp }(\mu \mathrm{m} \pm \mathrm{SD})$ & $3.96 \pm 0.34$ & $3.52 \pm 0.20$ \\
\hline $\mathrm{GSD}( \pm \mathrm{SD})$ & $2.25 \pm 0.05$ & $2.26 \pm 0.09$ \\
\hline $\mathrm{FPF}(\% \pm \mathrm{SD})$ & $17.5 \pm 2.0$ & $21.5 \pm 3.3$ \\
\hline $\mathrm{RF}(\% \pm \mathrm{SD})$ & $66.4 \pm 3.7$ & $73.2 \pm 6.9$ \\
\hline
\end{tabular}

FIG. 4. In vitro aerosol performance of DPPC/PLGA and PEI/DPPC/PLGA hNPs upon delivery through the Aeroneb ${ }^{\circledR}$ Go nebulizer. (A) Cumulative mass recovered as a function of the cutoff diameter of the NGI stages; (B) NGI deposition pattern; (C) fine particle characteristics of the aerosol cloud. Data are presented as mean \pm SD $(n=3)$. NGI, Next Generation Impactor. Color images available online at www.liebertpub.com/jamp

Nevertheless, while DPPC/PLGA Rhod $_{\text {formulation showed a }}$ peak at $5.39 \mu \mathrm{m}$, the highest percent of PEI/DPPC/PLGA ${ }_{\text {Rhod }}$ was recovered from cup with a cutoff diameter of $3.3 \mu \mathrm{m}$. As a consequence, by the same value of GSD, PEI/DPPC/PLGA Rhod hNPs displayed a lower $\mathrm{MMAD}_{\text {exp }}$ and, consequently, higher

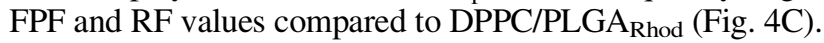

In vitro aerosolization of hNPS on TCCC

The ability of aerosolized hNPs to penetrate airway epithelial barrier was probed in vitro on TCCC. The experiments were carried out on fresh dispersions of freeze-dried hNPs (1:25 hNP/mannitol weight ratio) in $0.5 \mathrm{mM} \mathrm{NaCl}$ for ideal nebulization. Mannitol was added to the hNPs as cryoprotectant to avoid common hNP aggregation in a collapsed cake. ${ }^{(23)}$ In fact, it induced the lowest variation of $\mathrm{D}_{\mathrm{H}}$ and PI with respect to the control, and no difference was observed when changing the hNP/cryoprotectant ratio (Supplementary Table S1 and Supplementary Fig. S3). Furthermore, mannitol could also work facilitating hNP transport through mucus, and in so doing siRNA diffusion, due to its established ability to improve fluidity of mucus. ${ }^{(24)}$

The stability of hNPs during the nebulization process was confirmed by transmission electron microscopy (TEM) analysis of freeze-dried siRNA-loaded hNPs in the presence of mannitol (1:25 NP/mannitol ratio) before and after nebulization in the Vitrocell Cloud (Fig. 5). TEM images suggest a potential core-shell structure for both siRNA-DPPC/ PLGA and siRNA-PEI/DPPC/PLGA, which was retained after the nebulization process, which did not cause any apparent modification of the hNP structure or hNP aggregation for each tested concentration.

The deposition efficiency was also evaluated for both tested formulations, highlighting in each case a homogeneous distribution of the hNPs on the coverslips inside the exposure chamber (Supplementary Fig. S4) and a direct correlation between the delivered and deposited doses of hNPs. In particular, $0.0051 \pm 0.0018$ and $0.0127 \pm 0.0058 \mu \mathrm{g} / \mathrm{cm}^{2}$ of $\mathrm{hNPs}$ were deposited on coverslips after nebulization of DPPC/PLGA $\mathrm{Rhod}$ dispersions at low and high concentrations, respectively.

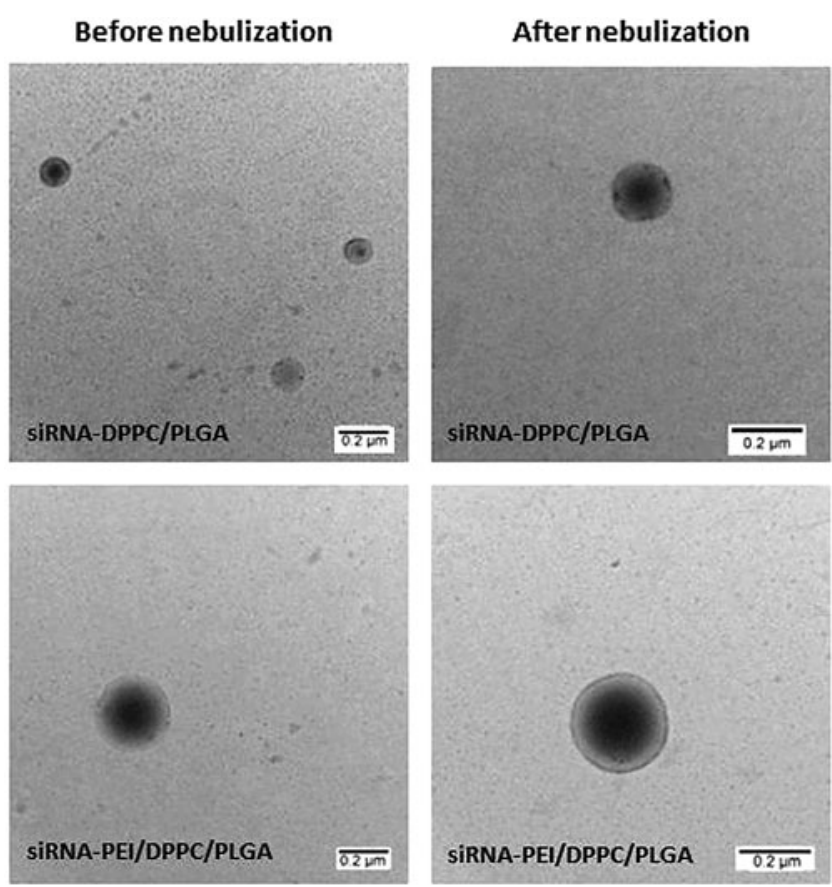

FIG. 5. Transmission electron microscopy (TEM) images of reconstituted freeze-dried siRNA-loaded hNPs (siRNADPPC/PLGA and siRNA-PEI/DPPC/PLGA) before and after nebulization. Scale bar is $0.2 \mu \mathrm{m}$. Field is representative of the sample. 

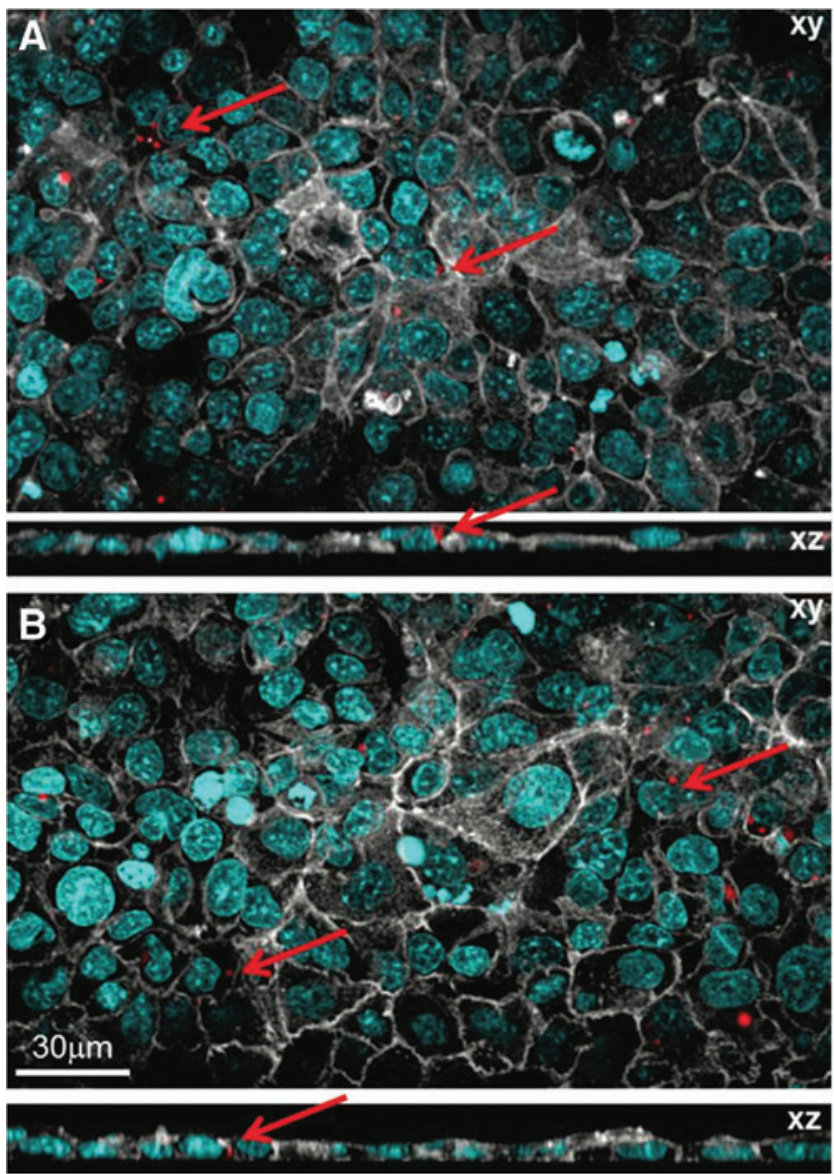

FIG. 6. CLSM images of TCCC after 24 hours postexposure to $0.5 \mathrm{mg}$ of fluorescently labeled DPPC/PLGA $\mathrm{Rhod}(\mathbf{A})$ and PEI/DPPC/PLGA Rhod $_{\text {(B) hNPs. Red color corresponds }}$ to the hNPs, white color to cytoskeletal F-actin, and cyan color to nulcei. The arrows show hNPs engulfed by the cells. TCCC, triple cell coculture model. Color images available online at www.liebertpub.com/jamp

Meanwhile, likely, as a consequence of the good aerosolization properties of PEI-containing formulations, $0.0137 \pm$ 0.0032 and $0.0334 \pm 0.0069 \mu \mathrm{g} / \mathrm{cm}^{2}$ of hNPs were recovered from coverslips after nebulization of low and high concentra-

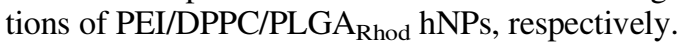

\section{Fate and cytotoxicity of aerosolized hNPs in TCCC}

To study the cell uptake, fluorescently labeled hNPs were aerosolized on TCCC. After 24 hours postexposure to hNPs, TCCC were visualized by CLSM (Fig. 6). Results indicate that both DPPC/PLGA Rhod $_{\text {and PEI/DPPC/PLGA }}$ Rhod hNPs (labeled in red) can effectively penetrate into cells and are localized intracellularly.

At the tested concentrations, the developed hNPs displayed no significant cytotoxicity compared to both sodium chloride and mannitol solutions aerosolized at the same concentrations of the samples $(p>0.5)$ (Fig. 7A). On the other hand, a significant cytotoxic effect was observed when the cocultures were incubated with Triton-X as positive control $(p<0.001$ versus DPPC/PLGA Rhod $_{\text {and PEI/DPPC/PLGA }}$ Rhod). These findings were confirmed by CLSM analysis of all treated
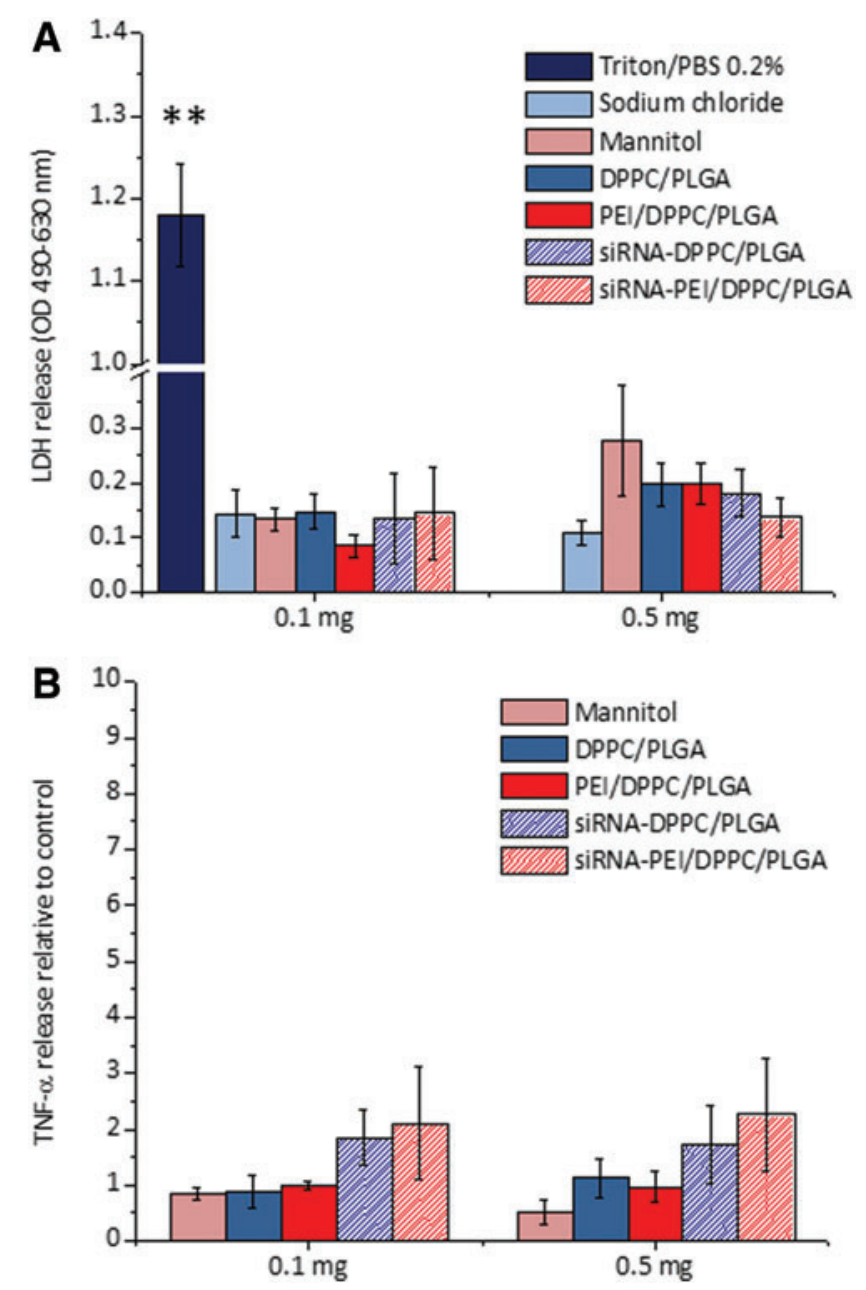

FIG. 7. LDH release (A) and TNF- $\alpha$ secretion (B) of TCCC after 24 hours postexposure to different theoretical amounts $(0.1$ and $0.5 \mathrm{mg})$ of aerosolized empty or siRNA-loaded hNPs. Sodium chloride was used as negative control. Mannitol aqueous solutions at the same volume and concentrations used for hNP samples were also aerosolized for comparison. Triton $0.2 \%$ (w/v) in PBS was used as positive control in LDH release (A). LDH data are presented as mean optical density (OD) values \pm SEM of samples diluted $1: 10 \mathrm{v} / \mathrm{v}$ in cell culture medium $(n=3)(* * p<0.001)$. TNF- $\alpha$ data are presented as the mean fold increase of samples relative to the negative control (i.e., sodium chloride \pm SEM $(n=3)$. LDH, lactate dehydrogenase; SEM, standard error mean; TNF- $\alpha$, tumor necrosis factor alpha. Color images available online at www.liebertpub.com/jamp

samples, since no alteration of cell morphology occurred after TCCC exposure to hNPs compared to the negative control (Supplementary Fig. S5).

The potential proinflammatory effect of aerosolized empty and siRNA-loaded hNPs on TCCC was also investigated by determining TNF- $\alpha$ secretion 24 hours postexposure (Fig. 7B). Of note, exposure to mannitol and empty hNPs, at both low and high concentration, caused a less than onefold increase of TNF$\alpha$ release to the TCCC exposed to $0.5 \mathrm{mM}$ aqueous sodium chloride. As for siRNA-loaded hNPs, we can see at all concentrations tested a higher production of TNF- $\alpha$ in comparison to negative control, although values were not significantly different from the mannitol control. 


\section{In vitro silencing effect of siRNA-loaded hNPs}

Once proven that the developed hNPs are able to overcome the lung barriers and reach the target cells, we further evaluated the effect of siRNA-loaded hNPs on gene silencing efficiency in a human lung epithelial cell line, that is A549 cells. Preliminary cytotoxicity studies showed that both DPPC/PLGA and PEI/DPPC/PLGA hNPs exhibited a safe profile toward A549 cells in a wide range of concentrations (from 0.2 to $2 \mathrm{mg} / \mathrm{mL}$ ) up to 72 hours of incubation (Supplementary Fig. S6). Nevertheless, to determine the amount of siRNA-loaded hNPs necessary to obtain an efficient gene depletion, dose-response studies were further performed by transiently transfecting A549 cells with increasing concentrations of siRNA pool by using lipofectamine. Results showed a specific and dose-dependent silencing of both $\alpha \mathrm{ENaC}$ and $\beta \mathrm{ENaC}$ subunits (Supplementary Fig. S7). In particular, while nontargeting siRNA pool at $20 \mathrm{nM}$ did not exert any effect on the levels of $\alpha \mathrm{ENaC}$ and $\beta \mathrm{ENaC}$ subunits (data not shown), siRNA pool against ENaC resulted in robust protein knockdown at 24 hours (about $50 \%$ and $60 \%$ of gene depletion for $\alpha \mathrm{ENaC}$ and $\beta \mathrm{ENaC}$, respectively). This siRNA concentration was thus selected for in vitro gene silencing studies with
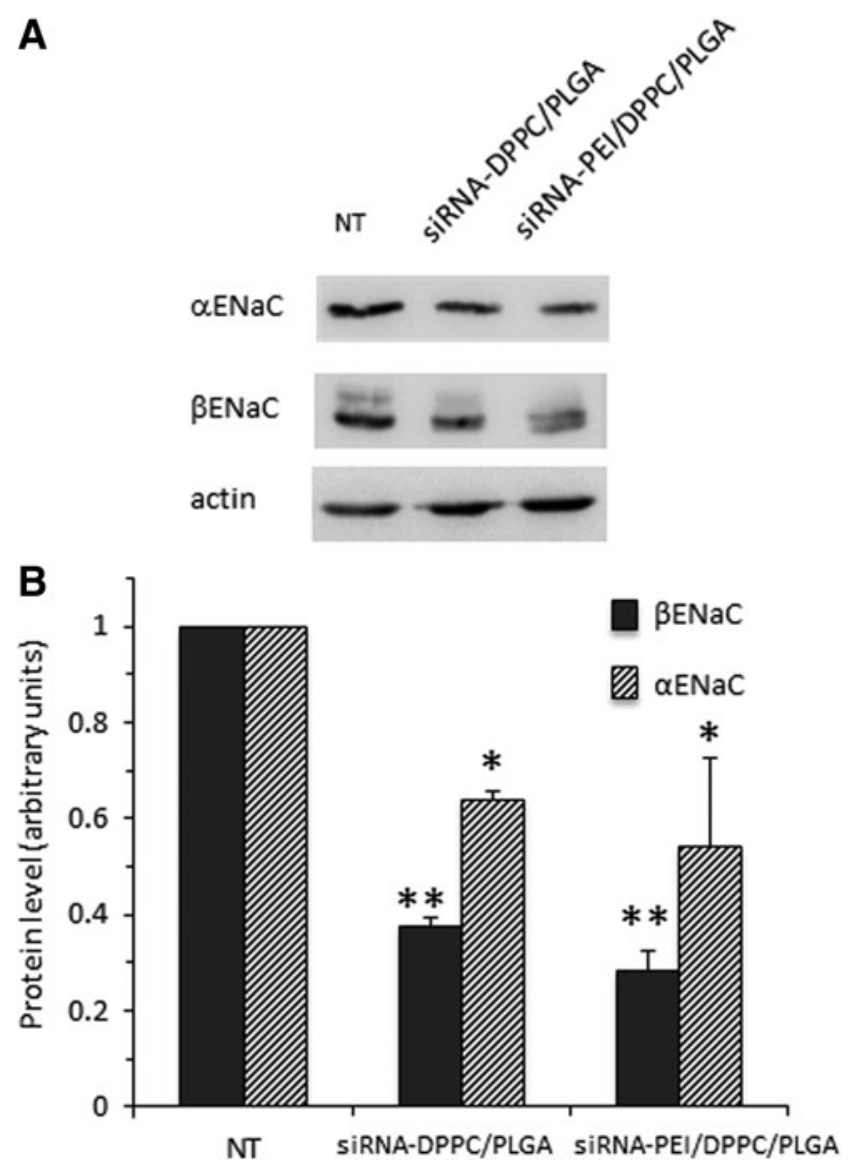

FIG. 8. Representative Western blotting of protein extracts from A549 cells treated or not with siRNA- DPPC/PLGA or siRNA-PEI/DPPC/PLGA for 72 hours. $\alpha \mathrm{ENaC}$ and $\beta \mathrm{ENaC}$ proteins were detected by using the indicated antibodies. Antiactin was used as the loading control (A). The quantification of signals is shown (B). The signal of untreated cells (NT) was set as 1 .
siRNA-loaded hNPs. We found that siRNA pool slowly released from hNPs resulted in efficient knockdown of both $\alpha \mathrm{ENaC}$ and $\beta \mathrm{ENaC}$ subunit proteins up to 72 hours (Fig. 8). Western blots confirmed that protein expression was significantly reduced by more than $60 \%$ and $40 \%$ for $\alpha \mathrm{ENaC}$ and $\beta \mathrm{ENaC}$, respectively, by siRNA-DPPC/PLGA hNPs compared to control (untreated cells). Of note, the addition of PEI inside the formulation increased the silencing efficiency of siRNAloaded hNPs by a further significant $10 \%$ compared to siRNADPPC/PLGA hNPs. The same amount of blank DPPC/PLGA and PEI/DPPC/PLGA hNPs did not affect the levels of ENaC subunits (data not shown).

\section{Discussion}

The importance of lung barriers in determining the therapeutic efficacy of inhaled drugs in severe lung diseases, such as $\mathrm{CF}$, is nowadays acknowledged. ${ }^{(7)}$ A current paradigm suggests that only NPs with appropriate size and surface properties may overcome the complex mucus airway barrier and assist drug interactions with human airway epithelial cells. ${ }^{(25,26)}$ This aspect is particularly crucial for siRNA-based therapeutics, the clinical application of which is further hindered by the short half-life in vivo and the low capability to penetrate cell membranes. To overcome these issues, we have designed and developed novel DPPC/PLGA hNPs, containing or not PEI as third component, suitable for pulmonary delivery of siRNA.

In line with literature data, ${ }^{(10)}$ a DPPC layer is likely present on the surface of PLGA particles giving rise to a core-shell structure, which is independent of the presence of PEI. As demonstrated by $\mathrm{hNP} /$ mucus interaction studies, this structure results in non-mucoadhesive or muco-inert hNPs, reasonably able to easily penetrate across the airway mucus layer. ${ }^{(8,17)}$ Furthermore, the adopted formulation conditions allowed the production of hNPs with a hydrodynamic diameter of $150 \mathrm{~nm}$, which are expected to easily diffuse through the gel-like structure of the mucus. ${ }^{(25,27)}$ Finally, aqueous hNPs were transformed into a solid long-term stable lyophilized powder, which can be reconstituted in saline and, likely, employed to co-administer a mucus rehydrating agent, that is mannitol. ${ }^{(24)}$

If DPPC, which is the principal component of the lung lining fluids, is expected to tune hNP interactions with the lung environment, the addition of PEI can play a crucial role for siRNA entrapment inside DPPC/PLGA hNPs. This hypothesis was confirmed by encapsulation studies performed on two siRNA sequences, for $\alpha$ and $\beta \mathrm{ENaC}$ subunits, which have been shown to produce a long-term reduction of transepithelial $\mathrm{Na}^{+}$currents and fluid absorption. ${ }^{(28)}$ In particular, the combination of the two siRNA can expand the lung fluid volume in vitro. ${ }^{(29)}$ Of note, siRNA was entrapped inside selected hNPs with high efficiency, particularly when PEI was coencapsulated. This effect could be likely ascribed to the formation of a complex between PEI, employed at the theoretical N/P ratio of 10, and siRNA.

Information about the presence of PEI/siRNA complexes inside hNPs could not be derived from SAXS spectra, due to the very low amounts of both components. Nevertheless, SAXS analysis clearly demonstrated that the role of PEI in the formulation is not to affect the overall particle morphology, except for a very slight decrease of the average size. On the other hand, the phospholipid component is possibly coating the PLGA particles, as suggested by TEM analysis. Furthermore, 
independent of the presence of PEI inside the formulation, hNPs were stable inside artificial CF mucus at least for 12 hours, confirming, along with in vitro aerosol performance studies, the potential of the system for direct aerosolization on the mucus-lined human airway epithelial barrier.

In fact, to attain maximum siRNA availability at lung epithelial cells, where its target is located, the hNPs should (i) uniformly deposit on the airway epithelium in their stable form; (ii) penetrate inside the lung epithelial barrier; and (iii) interact with the lung epithelium, without compromising cell availability. To draw key information on all these points and to establish a priori target formulation parameters for siRNA delivery to human airway epithelium, the behavior of the developed DPPC/PLGA hNPs after deposition on triple cell co-cultures, which realistically mimic human airway epithelium environment, was investigated.

When grown on porous supports at ALI, human bronchial epithelial cells reproduce in vivo morphology and key physiologic processes, sometimes including tight junctions and basal and mucus cells. ${ }^{(19,30)}$ Furthermore, direct deposition of the aerosolized drug to the apical compartment of the cell culture can be envisaged through systems especially designed for dose-controlled and spatially uniform deposition of liquid aerosols on cells, ${ }^{(31,32)}$ such as the Vitrocell Cloud employed in this study. Results showed that the adopted formulation conditions allowed to tune hNP properties, so as to achieve uniform and efficient aerosolization on lung epithelial cells in their integer form. Afterward, hNPs were demonstrated to penetrate inside extracellular lung lining fluids, to be taken up by lung epithelial cells without exerting a cytotoxic effect or a proinflammatory response on the treated lung epithelium model.

Once siRNA molecules overcome the aforementioned lung epithelial barriers and reach the target cells, they still need to overcome a series of intracellular barriers to exert their activity. Following cell entry, siRNA molecules must escape early from endosomes to avoid exposure to the noxious acidic environment as well as late-stage attack by the hydrolases in the endolysosome. ${ }^{(4)}$ Noteworthy, in vitro ENaC silencing studies ruled out any degradation process, while proving the ability of the siRNA released from hNPs to reach the cytoplasm and, after binding to its target mRNA, to silence the gene of interest. As a consequence, robust and long-lasting knockdown of both $\alpha \mathrm{ENaC}$ and $\beta \mathrm{ENaC}$ proteins could be achieved.

\section{Conclusions}

In this work, we have successfully designed and developed novel hNPs comprising PLGA likely surrounded by a DPPC shell, showing size and surface properties optimized for siRNA transport and delivery at lung. Optimized formulations allowed the encapsulation of a combination of siRNA against $\alpha$ and $\beta$ subunits of $\mathrm{ENaC}$ channel with high efficiency, which could be optimized by the addition of PEI inside the formulation. The developed hNPs were muco-inert and stable inside AM. Stable hNP-based dry powders were produced by freeze-drying with mannitol, an osmotic agent of interest for CF treatment. Cell uptake studies upon in vitro aerosolization on TCCC confirmed the ability of fluorescently labeled hNPs to be internalized inside the airway epithelial barrier. Hazard assessment of hNPs demonstrated that the developed system does not exert cytotoxic or acute proinflammatory effect toward any of the cell components of the co-culture model. Finally, in vitro gene silencing studies highlighted that released siRNAs are capable to reduce $\mathrm{ENaC}$ protein expression in human lung epithelial cells. Overall, results demonstrate the great potential of DPPC/ PLGA hNPs as carriers for siRNA delivery on the human epithelial airway barrier, prompting toward investigation of their therapeutic effectiveness in severe lung diseases.

\section{Acknowledgments}

F.U. gratefully acknowledges Compagnia di San Paolo and Istituto Banco di Napoli-Fondazione (STAR Program, Junior Principal Investigator Grant 2013, Napoli_call2013_35) for financial support. G.C. wishes to thank the International Society for Aerosols in Medicine (ISAM) for the 2015 ISAM Student Fellowships. E.D., A.P.F., and B.R.R. acknowledge the financial support of the Adolphe Merkle Foundation. The authors are grateful to Dr. T. Nrayanan, Dr. J. Moller, and to the ID02 beamline staff at the European Synchrotron Facility (Grenoble, France) and to Dr. Laetitia Haenni and Dr. Christoph Monnier at TEM facility of the Adolphe Merkle Institute for precious technical assistance.

\section{Author Disclosure Statement}

No competing financial interests exist.

\section{References}

1. Burnett JC, Rossi JJ, and Tiemann K: Current progress of siRNA/shRNA therapeutics in clinical trials. Biotechnol J. 2011;6:1130-1146.

2. Lee SH, Kang YY, Jang HE, and Mok H: Current preclinical small interfering RNA (siRNA)-based conjugate systems for RNA therapeutics. Adv Drug Deliv Rev. 2016; 104:78-92.

3. Karras JG, Sun G, Tay J, and Jackson AL: Reflections on microRNAs in chronic pulmonary disease: Looking into the miR-ror and crystal ball. Inflamm Allergy Drug Targets. 2013;12:88-98.

4. Lam JK, Liang W, and Chan HK: Pulmonary delivery of therapeutic siRNA. Adv Drug Deliv Rev. 2012;64:1-15.

5. Merkel OM, Rubinstein I, and Kissel T: siRNA delivery to the lung: What's new? Adv Drug Deliv Rev. 2014;75:112-128.

6. Ungaro F, d'Angelo I, Miro A, La Rotonda MI, and Quaglia F: Engineered PLGA nano- and micro-carriers for pulmonary delivery: Challenges and promises. J.Pharm Pharmacol. 2012;64:1217-1235.

7. d'Angelo I, Conte C, La Rotonda MI, Miro A, Quaglia F, and Ungaro F: Improving the efficacy of inhaled drugs in cystic fibrosis: Challenges and emerging drug delivery strategies. Adv Drug Deliv Rev. 2014;75:92-111.

8. d'Angelo I, Casciaro B, Miro A, Quaglia F, Mangoni ML, and Ungaro F: Overcoming barriers in Pseudomonas aeruginosa lung infections: Engineered nanoparticles for local delivery of a cationic antimicrobial peptide. Colloids Surf B Biointerfaces. 2015;135:717-725.

9. Griffiths PC, Cattoz B, Ibrahim MS, and Anuonye JC: Probing the interaction of nanoparticles with mucin for drug delivery applications using dynamic light scattering. Eur J Pharm Biopharm. 2015;97:218-222.

10. Raemdonck K, Braeckmans K, Demeester J, and De Smedt SC: Merging the best of both worlds: Hybrid lipid-enveloped 
matrix nanocomposites in drug delivery. Chem Soc Rev. 2014;43:444-472.

11. Pandita D, Kumar S, and Lather V: Hybrid poly(lactic-coglycolic acid) nanoparticles: Design and delivery prospectives. Drug Discov Today 2015;20:95-104.

12. Ungaro F, Giovino C, Coletta C, Sorrentino R, Miro A, and Quaglia F: Engineering gas-foamed large porous particles for efficient local delivery of macromolecules to the lung. Eur J Pharm Sci. 2010;41:60-70.

13. De Stefano D, Ungaro F, Giovino C, Polimeno A, Quaglia F, and Carnuccio R: Sustained inhibition of IL-6 and IL-8 expression by decoy ODN to NF-kappaB delivered through respirable large porous particles in LPS-stimulated cystic fibrosis bronchial cells. J Gene Med. 2011;13:200208.

14. Blank F, Rothen-Rutishauser B, and Gehr P: Dendritic cells and macrophages form a transepithelial network against foreign particulate antigens. Am J Respir Cell Mol Biol. 2007; 36:669-677.

15. Maiolino S, Moret F, Conte C, Fraix A, Tirino P, Ungaro F, Sortino S, Reddi E, and Quaglia F: Hyaluronan-decorated polymer nanoparticles targeting the CD44 receptor for the combined photo/chemo-therapy of cancer. Nanoscale. 2015; 7:5643-5653.

16. Narayanan T, Diat $O$, and B+Âsecke P: SAXS and USAXS on the high brilliance beamline at the ESRF. Nuclear Instruments and Methods in Physics Research Section A: Accelerators, Spectrometers, Detectors and Associated Equipment 2001;467-468, Part 2:1005-1009.

17. Ungaro F, d'Angelo I, Coletta C, d'Emmanuele di Villa Bianca R, Sorrentino R, Perfetto B, Tufano MA, Miro A, La Rotonda MI, and Quaglia F: Dry powders based on PLGA nanoparticles for pulmonary delivery of antibiotics: Modulation of encapsulation efficiency, release rate and lung deposition pattern by hydrophilic polymers. J Control Release. 2012;157:149-159.

18. Marple VA, Olson BA, Santhanakrishnan K, Roberts DL, Mitchell JP, and Hudson-Curtis BL: Next generation pharmaceutical impactor: A new impactor for pharmaceutical inhaler testing. Part III. extension of archival calibration to $15 \mathrm{~L} / \mathrm{min}$. J Aerosol Med. 2004;17:335-343.

19. Lehmann AD, Daum N, Bur M, Lehr CM, Gehr P, and Rothen-Rutishauser BM: An in vitro triple cell coculture model with primary cells mimicking the human alveolar epithelial barrier. Eur J Pharm Biopharm. 2011;77:398-406.

20a. Russo A, Saide A, Cagliani R, Cantile M, Botti G, and Russo G: rpL3 promotes the apoptosis of p53 mutated lung cancer cells by down-regulating $\mathrm{CBS}$ and $\mathrm{NF} \kappa \mathrm{B}$ upon 5-FU treatment. Sci Rep. 2016;6:38369.

20b. Pagliara V, Saide A, Mitidieri E, d'Emmanuele di Villa Bianca R, Sorrentino R, Russo G, and Russo A: 5-FU targets rpL3 to induce mitochondrial apoptosis via cystathionine- $\beta$-synthase in colon cancer cells lacking p53. Oncotarget. 2016;7:50333-50348.

21. De Filippis D, Russo A, De SD, Cipriano M, Esposito D, Grassia G, Carnuccio R, Russo G, and Iuvone T: Palmitoylethanolamide inhibits $\mathrm{rMCP}-5$ expression by regulating MITF activation in rat chronic granulomatous inflammation. Eur J Pharmacol. 2014;725:64-69.

22. Guinier G, and Fournet G: Small Angle Scattering of $X$ Rays. John Wiley \& sons, New York, 1955. pp. 126-160.

23. Fonte P, Reis S, and Sarmento B: Facts and evidences on the lyophilization of polymeric nanoparticles for drug delivery. J Control Release 2016;225:75-86.
24. Nolan SJ, Thornton J, Murray CS, and Dwyer T: Inhaled mannitol for cystic fibrosis. Cochrane Database Syst Rev. 2015;10:CD008649.

25. Ensign LM, Tang BC, Wang YY, Tse TA, Hoen T, Cone R, and Hanes J: Mucus-penetrating nanoparticles for vaginal drug delivery protect against herpes simplex virus. Sci Transl Med. 2012;4:138-179.

26. d'Angelo I, Conte C, Miro A, Quaglia F, and Ungaro F: Pulmonary drug delivery: A role for polymeric nanoparticles? Curr Top Med Chem. 2015;15:386-400.

27. Lai SK, Wang YY, Hida K, Cone R, and Hanes J: Nanoparticles reveal that human cervicovaginal mucus is riddled with pores larger than viruses. Proc Natl Acad Sci U S A. 2010;107:598-603.

28. Caci E, Melani R, Pedemonte N, Yueksekdag G, Ravazzolo R, Rosenecker J, Galietta LJ, and Zegarra-Moran O: Epithelial sodium channel inhibition in primary human bronchial epithelia by transfected siRNA. Am J Respir Cell Mol Biol. 2009;40:211-216.

29. Gianotti A, Melani R, Caci E, Sondo E, Ravazzolo R, Galietta LJ, and Zegarra-Moran O: Epithelial sodium channel silencing as a strategy to correct the airway surface fluid deficit in cystic fibrosis. Am J Respir Cell Mol Biol. 2013;49:445-452.

30. Rothen-Rutishauser BM, Kiama SG, and Gehr P: A threedimensional cellular model of the human respiratory tract to study the interaction with particles. Am J Respir Cell Mol Biol. 2005;32:281-289.

31. Endes C, Schmid O, Kinnear C, Mueller S, CamareroEspinosa S, Vanhecke D, Foster EJ, Petri-Fink A, RothenRutishauser B, Weder C, and Clift MJ: An in vitro testing strategy toward mimicking the inhalation of high aspect ratio nanoparticles. Part FibreToxicol. 2014;11:40.

32. Endes C, Mueller S, Kinnear C, Vanhecke D, Foster EJ, PetriFink A, Weder C, Clift MJ, and Rothen-Rutishauser B: Fate of cellulose nanocrystal aerosols deposited on the lung cell surface in vitro. Biomacromolecules. 2015;16:1267-1275.

Received on January 3, 2017 in final form, July 26, 2017

Reviewed by:

Olivia Merkel Sally-Ann Cryan

Address correspondence to: Barbara Rothen-Rutishauser, PhD Adolphe Merkle Institute University of Fribourg Chemin des Verdiers 4 Fribourg 1700 Switzerland

E-mail: barbara.rothen@unifr.ch

Francesca Ungaro, PhD Department of Pharmacy University of Napoli Federico II Via D. Montesano 49 Napoli 80131 Italy

E-mail: ungaro@unina.it 